cycles) or external radiotherapy (45-50 Gy total over 5-7 weeks).

At the median follow-up point of 95.5 months, 118 deaths had occurred -59 in the chemotherapy group and 59 in the radiotherapy group. Women in the chemotherapy group compared with the radiotherapy group had a nonsignificant hazard ratio for both death (0.95, $P=0.78)$ and first event (disease recurrence or death; $0.88, P=0.45$ ). At the third, fifth and seventh years of follow-up, overall survival was $76 \%, 66 \%$ and $62 \%$, respectively for patients in the chemotherapy group and $78 \%$, $69 \%$ and $62 \%$, respectively for patients in the radiotherapy group. Chemotherapy seemed to prevent or delay distant metastases more effectively than radiotherapy, while radiation therapy seemed to achieve better locoregional control; however, these trends did not reach statistical significance. On the basis of this observation, the authors suggest that the two approaches could be used concurrently or sequentially in an adjuvant setting, and in this regard trials comparing combined radiotherapy and chemotherapy with radiotherapy alone are of great interest.

Original article Maggi R et al. (2006) Adjuvant chemotherapy vs radiotherapy in high-risk endometrial carcinoma: results of a randomised trial. Br JCancer 95: 266-271

\section{High EGFR copy number predicts poor outcome in head and neck squamous cell carcinoma}

The EGFR gene is overexpressed in several forms of cancer. EGFR protects cancer cells from the cytotoxic effects of chemotherapy and radiotherapy, and EGFR inhibitors have a clinical benefit in a subset of patients with head and neck squamous cell carcinoma (HNSCC), increasing their response to radiotherapy and improving survival. Elucidating the EGFR pathway and identifying biomarkers for the disease will be crucial for predicting the treatment response to EGFR inhibitors and for patient selection.

Chung et al. analyzed 86 tumor samples from 82 patients with HNSCC. Fluorescence in situ hybridization (FISH), polymerase chain reaction and DNA sequence analysis, and DNA microarray analysis and immunohistochemistry, were used to determine the EGFR gene copy number, EGFR gene mutation, and EGFR RNA and protein expression, respectively. HNSCC patients with FISH-positive (EGFR high polysomy and/or gene amplification) tumors had shorter progression-free and overall survival than those with FISH-negative tumors $(P<0.05$ and $P<0.01$, respectively). No mutations in EGFR were found, but the expression of EGFR-coamplified and overexpressed protein (Ecop), which regulates the activity of nuclear factor kappa B, was increased in FISH-positive tumors. An increase in nuclear factor kappa B signaling is a marker of worse prognosis in HNSCC.

Although this study demonstrated an association between increased EGFR copy number and worse prognosis in HNSCC, the underlying molecular mechanism is unclear.

Original article Chung $\mathrm{CH}$ et al. (2006) Increased epidermal growth factor receptor gene copy number is associated with poor prognosis in head and neck squamous cell carcinomas. JClin Oncol 24: 4170-4176

\section{Assay differentiates lung adenocarcinoma from mesothelioma}

Malignant pleural effusion (MPE) is a common feature of both lung adenocarcinoma and malignant mesothelioma. Distinguishing between the two conditions can be difficult, as they have many clinical and histological similarities. Researchers in Australia have now developed a molecular diagnostic test for differentiating between adenocarcinoma and mesothelioma by gene-expression profiling of MPE samples.

After excluding genes expressed in normal mesothelial cells, Holloway et al. identified 17 genes that are differentially expressed between lung adenocarcinoma and mesothelioma. A quantitative real-time polymerase-chainreaction-based assay of gene expression in MPE samples was able to distinguish lung adenocarcinoma from mesothelioma in 15 of 16 cases; validation of the test in formalin-fixed paraffin-embedded MPE and lung biopsy samples from 13 patients ( 8 mesothelioma, 5 adenocarcinoma) showed that the gene-expression assay correctly predicted the diagnosis in all cases.

The authors conclude that the geneexpression assay can accurately differentiate lung adenocarcinoma from mesothelioma in both fresh-frozen and formalin-fixed pathology specimens of MPE. The test could avoid the 